\title{
Genetic analysis of the bacterial hook-capping protein FlgD responsible for hook assembly
}

\begin{abstract}
Correspondence
Tohru Minamino

tohru@fbs.osaka-u.ac.jp

Keiichi Namba

keiichi@fbs.osaka-u.ac.jp
\end{abstract}

Received 24 November 2010

Revised 16 January 2011

Accepted 19 February 2011

\author{
Nao Moriya, ${ }^{1} \dagger$ Tohru Minamino, ${ }^{1,2} \dagger$ Katsumi Imada ${ }^{1,3}$ and Keiichi Namba ${ }^{1}$ \\ ${ }^{1}$ Graduate School of Frontier Biosciences, Osaka University, 1-3 Yamadaoka, Suita, \\ Osaka 565-0871, Japan \\ ${ }^{2}$ PRESTO, JST, 4-1-8 Honcho, Kawaguchi, Saitama 332-0012, Japan \\ ${ }^{3}$ Department of Macromolecular Science, Graduate School of Science, Osaka University, \\ 1-1 Machikaneyama-cho, Toyonaka, Osaka 560-0043, Japan
}

FlgD of Salmonella enterica is a 232 aa protein that acts as the hook cap to promote assembly of FlgE into the hook structure. The $\mathrm{N}$-terminal 86 residues $\left(\mathrm{FlgD}_{\mathrm{N}}\right)$ complement $\mathrm{flg} D$ mutants, albeit to a small degree. However, little is known about the role of the C-terminal region of $\mathrm{FlgD}\left(\mathrm{FlgD}_{\mathrm{C}}\right)$. Here we isolated pseudorevertants from Salmonella flgE mutants. About half of the extragenic mutations lay within $\mathrm{FlgD}_{\mathrm{C}}$ and only one in $\mathrm{FlgD}_{\mathrm{N}}$. These suppressor mutations prevented mutant FlgE subunits from leaking out to some degree. Two weakly motile flgD mutants encoding C-terminally truncated variants, $\mathrm{FlgD}_{(1-195)}$ and $\mathrm{FlgD}_{(1-138 \mathrm{f}-\mathrm{s}+4 \mathrm{aa})}$, secreted larger amounts of $\mathrm{FlgE}$ into the culture medium than wild-type cells. Their hooks were shorter, and their length distributions were broader, with significant tailing towards smaller values. These results suggest that $\mathrm{FlgD}_{\mathrm{C}}$ contributes to efficient hook polymerization. Therefore, we propose that $\mathrm{FlgD}_{\mathrm{N}}$ attaches to the distal end of the hook to promote hook polymerization and that $\mathrm{FlgD}_{\mathrm{C}}$ blocks the exit of newly exported FlgE monomers into the culture medium, allowing FlgE to have more time to assemble into the hook.

\section{INTRODUCTION}

The flagellum drives bacteria through their environments. The flagellum of Salmonella enterica is divided into at least five substructures: the basal body, the hook, the hookfilament junction zone, the filament and the filament cap. The basal body, which consists of the $\mathrm{C}$ ring (FliG, FliM and $\mathrm{FliN}$ ), the $\mathrm{MS}$ ring (FliF), the $\mathrm{P}$ ring (FlgI), the $\mathrm{L}$ ring $(\mathrm{FlgH})$ and the rod ( $\mathrm{FliE}, \mathrm{FlgB}, \mathrm{FlgC}, \mathrm{FlgF}$ and $\mathrm{FlgG}$ ), is embedded in the cell surface and functions as a rotary motor. The hook (FlgE) and filament (FliC) extend from the exterior of the cell. The filament is a helical tube structure and works as a screw. The hook connects the rod to the filament and functions as a universal joint to smoothly transmit torque produced by the motor to the filament. The hook-filament junction zone (FlgK and FlgL) is located between the hook and filament, and acts as a buffering structure connecting these two filamentous structures with distinct mechanical characteristics. The filament cap is located at the tip of the filament to promote filament formation (Macnab, 2003; Minamino \& Namba, 2004).

†These authors contributed equally to this work.

A supplementary figure, showing a sequence alignment of FlgD from Salmonella enterica and Xanthomonas campestris, is available with the online version of this paper.
Flagellar assembly begins with the basal body, followed by the hook, and finally the filament. For construction of the flagellum, most of the flagellar proteins are delivered to the distal end of the growing structure by the flagellar type III protein export apparatus (Minamino \& Macnab, 1999). Three capping proteins, FlgJ, FlgD and FliD, are required for polymerization of the rod, the hook and the filament, respectively (Ikeda et al., 1985; Ohnishi et al., 1994; Hirano et al., 2001). The FliD cap is made of a pentagonal plate domain as a lid and five axially extended leg-like domains that bind to the filament at the distal end, forming a cavity under the pentagonal plate as a folding chamber for newly exported FliC molecules (Yonekura et al., 2000). There is a symmetry mismatch between the helical subunit array of the filament, with 11 protofilaments forming the tube and the pentameric annular structure of the FliD cap, and this symmetry mismatch is the basis of the mechanism of assembly promotion by the FliD cap (Yonekura et al., 2000).

The hook is a highly curved tubular structure composed of about 120 subunits of FlgE, and assembles with a helical symmetry. FlgE can polymerize in vitro onto the tip of the preexisting hook (Kato et al., 1982). In contrast, FlgD helps the attachment of FlgE monomers to the distal end of the rod and the growing hook (Kubori et al., 1992). When purified FlgD is supplied to $f l g D$ mutants, the hook grows 
and hence the mutants recover motility. This suggests that FlgD permits the incorporation of newly exported FlgE monomers to the hook tip in a way similar to the FliD cap (Ohnishi et al., 1994). FlgD is located at the distal end of the hook isolated from $f l g K$ mutants but is no longer present at the tip of the hook prepared from flgL mutants, indicating that the FlgD cap is displaced by FlgK prior to filament formation (Ohnishi et al., 1994). Thus, the FlgD cap is only transiently associated with the tip of the hook during hook formation.

The length of the hook is controlled at about $55 \mathrm{~nm}$ (Hirano et al., 1994), and FlhB and FliK are involved in hook length control. FlhB is one of the integral membrane proteins of the export apparatus (Minamino \& Macnab, 2000). FliK is thought to act as a ruler to measure the hook length (Moriya et al., 2006; Erhardt et al., 2010). When the hook has reached its mature length of $55 \mathrm{~nm}$, temporal association of the $\mathrm{N}$-terminal ruler domain of FliK with the inner surface of the hook during FliK secretion allows the C-terminal domain of FliK to interact with FlhB, thereby terminating the export of proteins required for assembly of the rod and hook, and initiating the export of proteins responsible for filament formation (Minamino et al., 2009).

Salmonella FlgD consists of 232 amino acid residues. FlgD has two domains: a flexible $\mathrm{N}$-terminal domain $\left(\mathrm{FlgD}_{\mathrm{N}}\right)$ and a compactly folded C-terminal domain $\left(\mathrm{FlgD}_{\mathrm{C}}\right.$ ) (Kuo et al., 2008). Kutsukake \& Doi (1994) have reported that the Nterminal 86 residues of Salmonella FlgD are functional as the hook cap. A partial atomic model of the hook has been built by the complementary use of X-ray crystallography and electron cryomicroscopy (Samatey et al., 2004; Fujii et al., 2009). Although the crystal structure of the C-terminal domain of FlgD from Xanthomonas campestris has been solved at $2.5 \AA$ (Kuo et al., 2008), how FlgD works is still unknown.

In this study, we carried out genetic analysis of FlgD by isolating suppressor mutants of $f l g E$ mutants that cannot form the hook structure at $42{ }^{\circ} \mathrm{C}$, characterized Cterminally truncated variants of $\mathrm{FlgD}$, and showed that $\mathrm{FlgD}_{\mathrm{C}}$ contributes to hook assembly. Based on these results, we discuss the role of $\mathrm{FlgD}_{\mathrm{C}}$ in hook assembly.

\section{METHODS}

Bacterial strains, plasmids, P22-mediated transduction, DNA manipulations and media. Bacterial strains and plasmids used in this study are listed in Table 1. P22-mediated transduction was carried out as described by Yamaguchi et al. (1984). Procedures for DNA manipulation were carried out as described previously (SaijoHamano et al., 2004). L broth (LB) and soft agar plates were prepared as described previously (Minamino \& Macnab, 1999). Ampicillin was added at a final concentration of $100 \mu \mathrm{g} \mathrm{ml}^{-1}$.

Motility assay on soft agar plates. Fresh colonies were inoculated onto soft agar plates and incubated at 30 or $42{ }^{\circ} \mathrm{C}$. At least seven independent experiments were performed.
Secretion assay. Salmonella cells were grown at 30 or $42{ }^{\circ} \mathrm{C}$ with shaking until the cell density had reached $\mathrm{OD}_{600} \sim 1.2-1.4$. After centrifugation, the cellular and culture supernatant fractions were collected separately. Cell pellets were resuspended in SDS-loading buffer and normalized with respect to the cell density to give a constant amount of cells. Proteins in the culture supernatants were precipitated by $10 \%$ TCA, suspended in a Tris/SDS loading buffer and heated at $95{ }^{\circ} \mathrm{C}$ for $5 \mathrm{~min}$. After SDS-PAGE, immunoblotting with polyclonal anti-FlgD, anti-FlgE, anti-FliK and anti-FliC antibodies was carried out as described previously (Minamino \& Macnab, 1999). The polyclonal anti-FlgD antibody efficiently recognizes the C-terminal portion of $\mathrm{FlgD}$, and hence the band of $\mathrm{FlgD}_{(1-138 \mathrm{f}-\mathrm{s}+4 \mathrm{aa})}$ was much weaker than those of full-length $\mathrm{FlgD}$ and $\mathrm{FlgD}_{(1-195)}$ (data not shown). At least three independent secretion assays were performed.

Measurement of hook length. The hook basal bodies were prepared as described by Aizawa et al. (1985), with minor modifications. Samples were negatively stained at $4{ }^{\circ} \mathrm{C}$ with $3 \%$ phosphotungstic acid ( $\mathrm{pH} 4.5$ ) on carbon-coated copper grids. Micrographs were recorded at a magnification of $\times 50000$ with a JEM-1011 transmission electron microscope (JEOL) operated at $100 \mathrm{kV}$. Hook length was measured using National Institutes of Health (NIH) Image 1.63 software. More than 200 hook basal bodies prepared from each Salmonella strain were measured.

\section{RESULTS}

\section{Isolation of pseudorevertants from hook assembly-deficient flgE mutants}

To investigate the hook assembly mechanism in more detail, we carried out genetic analyses of hook assemblydeficient flgE mutants. To do this, we first looked for hook assembly-deficient $f l g E$ point mutants from our collections. We previously identified eight weakly motile flgE mutants with lowered efficiency of hook assembly at $30{ }^{\circ} \mathrm{C}$ (Moriya et al., 2006). During further characterization of these mutants, we found that motility of the $f l g E(\mathrm{R} 96 \mathrm{~S})$, $f \lg E(\mathrm{I} 137 \mathrm{~S})$ and $f \lg E(\mathrm{~T} 149 \mathrm{I})$ mutants was much weaker than that of wild-type cells at $42{ }^{\circ} \mathrm{C}$, although slightly better than that of a completely assembly-defective $f \lg E(\Delta 9-20)$ mutant (Fig. 1a). Immunoblotting with polyclonal anti-FlgE antibody revealed that neither protein stability nor secretion of these point mutant FlgE proteins was affected at $42{ }^{\circ} \mathrm{C}$ (Fig. 1b, upper panel).

These flgE point mutants produce flagellar filaments at $30{ }^{\circ} \mathrm{C}$ (Moriya et al., 2006). Therefore, to test whether these mutants also produce the filaments even at $42{ }^{\circ} \mathrm{C}$, we analysed the cellular and secretion levels of FliC (Fig. 1b, lower panel). A huge amount of FliC was detected in both cell (lane 1) and culture supernatant fractions from wildtype cells (lane 6). However, only very faint FliC bands were seen in the two fractions from the flgE mutants (lanes 2-5 and 7-10). Consistently, the mutants produced the basal bodies, occasionally with very short hooks attached (Fig. 1c). These results suggest that they are severely defective in hook assembly at $42{ }^{\circ} \mathrm{C}$.

The crystal structure of FlgE31, a major proteolytic fragment of FlgE lacking both unfolded $\mathrm{N}$ - and C-terminal 
Table 1. Strains and plasmids used in this study

\begin{tabular}{|c|c|c|}
\hline Strain or plasmid & Relevant characteristics & Source or reference \\
\hline \multicolumn{3}{|l|}{ Salmonella strains } \\
\hline SJW1103 & Wild-type strain for motility and chemotaxis & Yamaguchi et al. (1984) \\
\hline SJW156 & $f \lg D(72 \mathrm{f}-\mathrm{s}+1 \mathrm{aa})$ & Ohnishi et al. (1994) \\
\hline SJW1353 & $f \lg E \Delta(9-20)$ & Ohnishi et al. (1994) \\
\hline SJW2236 & $f \lg E(\mathrm{~T} 149 \mathrm{I})$ & Moriya et al. (2006) \\
\hline SJW2248 & flgE(R96S) & Moriya et al. (2006) \\
\hline SJW2252 & $\operatorname{flg} E(\mathrm{I} 137 \mathrm{~S})$ & Moriya et al. (2006) \\
\hline MMD623 & $f \lg D\left(\mathrm{Q} 196^{*}\right)$ & This study \\
\hline MMD651 & $f l g D(138 \mathrm{f}-\mathrm{s}+4 \mathrm{aa})$ & This study \\
\hline MME2236-xx series & Pseudorevertants from SJW2236 & This study \\
\hline MME2248-xx series & Pseudorevertants from SJW2248 & This study \\
\hline MME2252-xx series & Pseudorevertants from SJW2252 & This study \\
\hline \multicolumn{3}{|l|}{ Plasmids } \\
\hline pRCD001 & pTrc99FF4/FlgD & Hirano et al. (2003) \\
\hline pMM701 & pTrc99AFF4/FlgD $(1-195)$ & This study \\
\hline pMM702 & pTrc99AFF4/FlgD ${ }_{(1-138 f-s+4 a a)}$ & This study \\
\hline pMM704 & pTrc99AFF4/FlgD $(1-86)$ & This study \\
\hline pNM001 & pTrc99AFF4/FlgE & Moriya et al. (2006) \\
\hline pNM003 & pTrc99AFF4/FlgE(T149I) & Moriya et al. (2006) \\
\hline
\end{tabular}

regions, has two domains, D1 and D2, connected by a short stretch of two-stranded anti-parallel $\beta$-sheet (Samatey et al., 2004). The R96S and I137S mutations are in domain D1, while the T149I mutation is in domain D2 (Fig. 1d). The side chains of Ile-137 and Thr-149 point to the domain cores of D1 and D2, respectively. The side chain of Arg-96 is exposed on the domain surface, but is not involved in the interaction with the neighbouring subunits. Therefore, we (a)

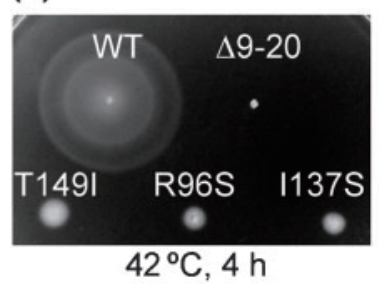

(c)

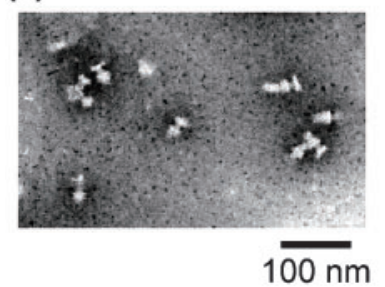

(b)

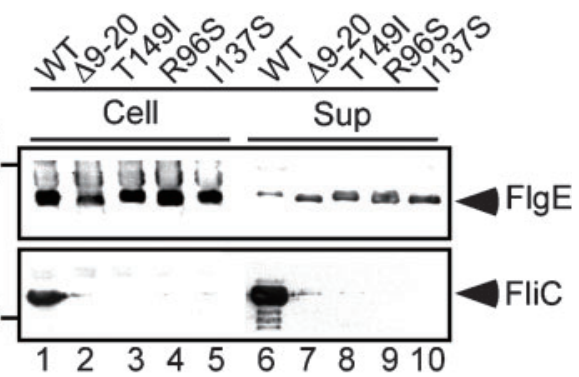

(d)

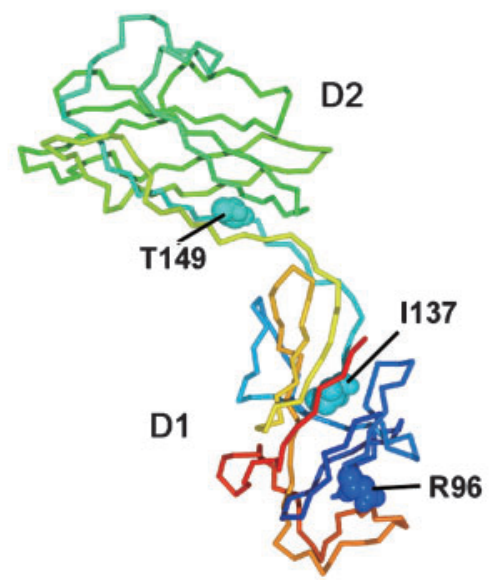

Fig. 1. Characterization of severely impaired motile flgE mutants. (a) Motility assay of SJW1103 (wild-type; WT) and four flgE mutants: SJW1353 ( $\Delta 9-20), \quad$ SJW2236 (T149I), SJW2248 (R96S) and SJW2252 (I137S). The soft agar plates were incubated at $42{ }^{\circ} \mathrm{C}$ for $4 \mathrm{~h}$. (b) Secretion of FlgE and FliC. Immunoblotting using polyclonal anti$\mathrm{FlgE}$ and anti-FliC antibodies of whole-cell proteins (Cell) and culture supernatant fractions (Sup) prepared from SJW1103 (WT), SJW1353 ( $\Delta 9-20), \quad$ SJW2236 (T149I), SJW2248 (R96S) and SJW2252 (I137S) grown at $42{ }^{\circ} \mathrm{C}$. The positions of $\mathrm{FlgE}$ and $\mathrm{FliC}$ are indicated on the right. Molecular mass markers $(\mathrm{kDa})$ are shown on the left. (c) Electron micrograph of flagellar structures isolated from SJW2236 grown at $42{ }^{\circ} \mathrm{C}$. (d) Crystal structure of the $31 \mathrm{kDa}$ fragment of Salmonella FlgE, which consists of two domains, D1 and D2 [Protein Data Bank (PDB) ID: 1WLG]. The $\mathrm{C} \alpha$ backbone is colour-coded from blue to red, going through the rainbow colours from the $\mathrm{N}$ to the $\mathrm{C}$ terminus. Point mutation sites that cause a defect in hook assembly are indicated by a CPK representation. 
assume that the effects of the R96S, I137S and T149I mutations may indirectly propagate to the subunit interface at $42{ }^{\circ} \mathrm{C}$.

To investigate the effect of these flgE mutations on hook assembly at $42{ }^{\circ} \mathrm{C}$, we isolated pseudorevertants from these flgE mutants by streaking out overnight cultures on soft agar plates, incubating them at $42{ }^{\circ} \mathrm{C}$ for 2 days and looking for motility haloes. Eight, five and 11 motile colonies were isolated from the $f l g E(\mathrm{R} 96 \mathrm{~S}), \mathrm{flg} E(\mathrm{I} 137 \mathrm{~S})$ and flgE(T149I) mutants, respectively. The motility of these pseudorevertants was clearly stronger than that of their parental flgE strains, although still poorer than that of wildtype cells (Fig. 2a).

DNA sequencing identified 24 missense mutations lying within $\mathrm{FlgD}$, FlgE or FlgG. The flgE(R96S) mutant gave rise to one intragenic suppressor, D63E; four extragenic suppressors in FlgD, K45R and T122R (isolated three times); and three extragenic suppressors in FlgG, Q121L (isolated twice) and G163V. The flgE(I137S) mutant gave rise to two intragenic suppressors, A135V (isolated twice); and three extragenic suppressors in FlgD, G105D, F125S and I153N. The $f l g E($ T149I) mutant gave rise to five intragenic suppressors in FlgE, T149S, A135V, D288Y and N303K (isolated twice); and six extragenic suppressors in FlgD, G100D (isolated twice), T122P, P124Q (isolated twice) and G167C. These second-site flgD mutations were all within $\mathrm{FlgD}_{\mathrm{C}}$, except for $\mathrm{FlgD}(\mathrm{K} 45 \mathrm{R})$, which lay within $\mathrm{FlgD}_{\mathrm{N}}$ (Fig. 2b).

We next tested whether these extragenic flgD suppressors display allele specificity. The $f l g D(\mathrm{~K} 45 \mathrm{R})$ and $f \lg D(\mathrm{~T} 122 \mathrm{R})$ mutations suppressed their parental $f \lg E(\mathrm{R} 96 \mathrm{~S})$ mutation but not the $f \lg E(\mathrm{~T} 149 \mathrm{I})$ mutation. The $f l g D(\mathrm{G} 100 \mathrm{D})$ and $f \lg D(\mathrm{~T} 122 \mathrm{P})$ mutations suppressed their parental $f l g E(\mathrm{~T} 149 \mathrm{I})$ mutation but not the $f l g E(\mathrm{R} 96 \mathrm{~S})$ mutation. Thus, these second-site $f l g D$ mutations did not give the pseudorevertant phenotype to other flgE mutants. These results suggest a possible interaction between $\mathrm{FlgD}_{\mathrm{C}}$ and FlgE.

To test the effect of each second-site mutation by itself on motility, we isolated the second-site flgD mutants and analysed their motility in soft agar. The motility of all mutants was normal at both 30 and $42{ }^{\circ} \mathrm{C}$ (data not shown). As these second-site mutations display no phenotype, they do not appear to affect the function of the wild-type FlgD protein.

\section{Effect of extragenic flgD suppressors on the secretion level of FlgE}

Large amounts of FlgE were seen in the culture supernatant of the parental flgE mutants compared with the wild-type (Fig. 1). Therefore, we tested whether the extragenic suppressor mutations in FlgD block the leakage of mutant FlgE monomers into the culture medium at $42{ }^{\circ} \mathrm{C}$. The amounts of FlgE secreted by the pseudorevertants were less


Fig. 2. Isolation of pseudorevertants from hook assembly-deficient flgE mutants. (a) Motility of SJW1103 (wild-type; WT), SJW2236 [flgE(T149l)], MME2236-9 [flgE(T149I)], $f / g D(G 167 C)$ and MME2236-12 [flgE(T149I/A135V)] in soft agar. The plate was incubated at $42{ }^{\circ} \mathrm{C}$ for $5 \mathrm{~h}$. (b) Schematic domain diagram of Salmonella hook-capping protein $\mathrm{FlgD}$ and its mutant variants, SJW156, MMD623 and MMD651. Locations of extragenic suppressor mutations of hook assembly-deficient flgE mutants are indicated by arrows. $\mathrm{N}$ and $\mathrm{C}$ indicate the $\mathrm{N}$ and $\mathrm{C}$ termini of FlgD, respectively. (c) Secretion of FlgE. Immunoblotting using polyclonal anti-FlgE antibody of whole-cell proteins (Cell) and culture supernatant fractions (Sup) prepared from SJW1103, SJW2236 and MME2236-9 grown at $42{ }^{\circ} \mathrm{C}$. The position of FlgE is indicated on the right. Molecular mass markers are shown on the left.

than those of their parental flgE mutants but still higher than the wild-type level (Fig. 2c). This suggests that the second-site mutations alter the conformation of FlgD to some degree to prevent mutant $\mathrm{FlgE}$ monomers from leaking out so that mutant FlgE molecules can have more time to be incorporated into the hook. 


\section{Characterization of weakly motile flgD mutants}

The N-terminal 86 residues of Salmonella FlgD are reported to be sufficient to complement $f l g D$ mutants (Kutsukake \& Doi, 1994). However, we found that most of the extragenic flgE suppressors were in $\mathrm{FlgD}_{\mathrm{C}}$, raising the possibility that $\mathrm{FlgD}_{\mathrm{C}}$ contributes to the hook assembly process. Therefore, we re-examined whether $\mathrm{FlgD}_{(1-86)}$ complements a hook assembly-defective $f l g D$ mutant strain, SJW156 [flg $D_{(1-72 f-}$ $s+1 a a)$, in which a single-base deletion was encountered at A215, resulting in a frame-shift mutation in FlgD (Fig. 2b). Wild-type FlgD restored full motility of the $f l g D_{(1-72 f-s+1 a a)}$ mutant (Fig. 3a). In contrast, $\mathrm{FlgD}_{(1-86)}$ restored motility to only a small degree (Fig. 3a). FliC was also detected in the culture supernatants, although at a much lower level than in the wild-type (Fig. 3b, upper panel). Consistently, the number of flagellar filaments was decreased, as judged by dark-field microscopy (data not shown). The amount of FlgE secreted by the cells expressing $\mathrm{FlgD}_{(1-86)}$ was much higher than that secreted by the wild-type (Fig. 3b, lower panel). Since $f l g D$ null mutants cannot produce the hook due to loss of the hook cap, large amounts of unassembled FlgE subunits are secreted into the periplasm and culture medium (Ohnishi et al., 1994; Minamino \& Macnab, 1999). Therefore, we suggest that $\mathrm{FlgD}_{\mathrm{N}}$ alone cannot exert the hook-capping function efficiently. (a)

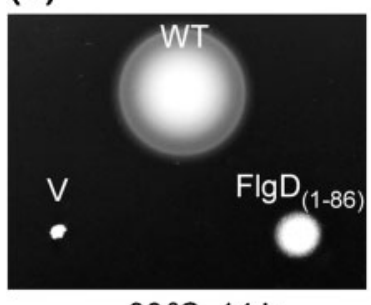

$30^{\circ} \mathrm{C}, 14 \mathrm{~h}$



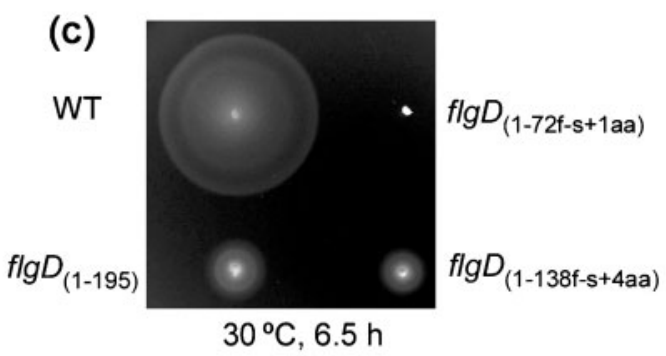

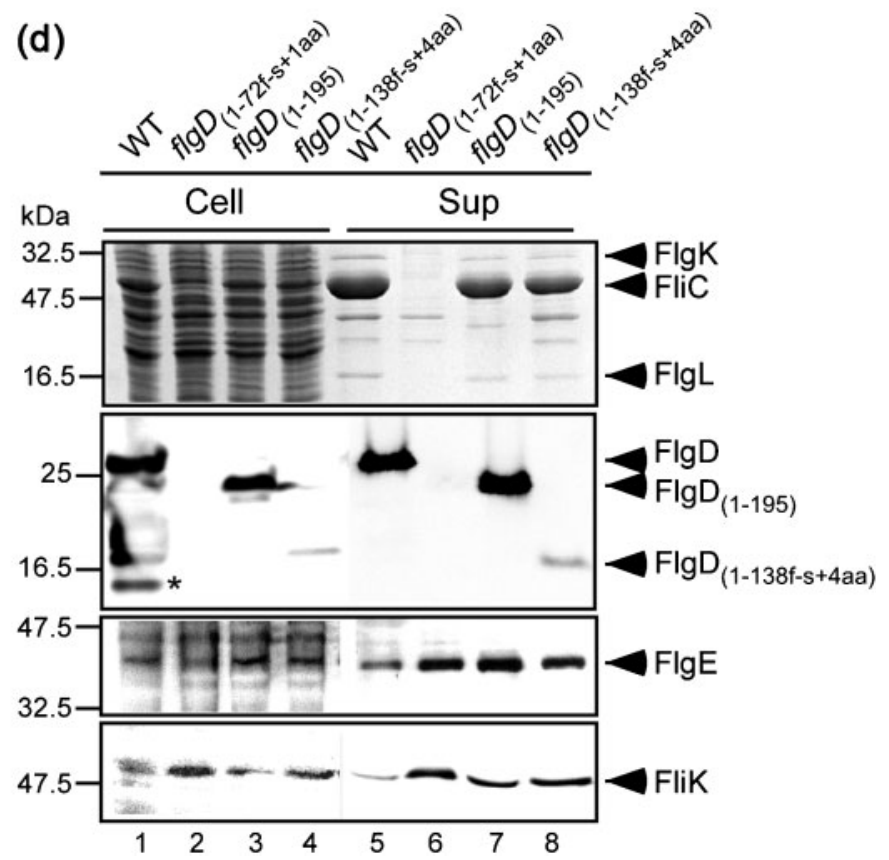

Fig. 3. Effect of C-terminal truncations of FlgD on motility. (a) Motility of SJW156 $\left[f / g D_{(72 f-s+1 a a)}\right]$ transformed with pTrc99A (V), pRCD001 (wild-type; WT) or pMM704 $\left[\mathrm{FlgD}_{(1-86)}\right]$ in soft agar. Plates were incubated at $30{ }^{\circ} \mathrm{C}$ for $14 \mathrm{~h}$. (b) Secretion of $\mathrm{FliC}$ and FlgE. Coomassie stained SDS gels (upper panel) and immunoblots with polyclonal antiFlgE antibody (lower panel) of whole-cell lysates (Cell) and culture supernatants (Sup) prepared from the same transformants as in (a). The positions of FliC and FlgE are indicated by arrowheads. Molecular mass markers are shown on the left. (c) Motility assay of SJW1103 (WT), SJW156 $\left[f \lg D_{(1-72 f-s+1 a a)}\right], \quad M M D 623\left[f l g D_{(1-195)}\right]$ and MMD651 $\left[f / g D_{(1-138 f-s+4 a a)}\right]$. Plates were incubated at $30{ }^{\circ} \mathrm{C}$ for $6.5 \mathrm{~h}$. (d) Secretion assays. Secretion levels of $\mathrm{FliC}, \mathrm{FlgK}$ and FlgL were analysed by Coomassie brilliant blue staining (first row), while those of FlgD, FlgE and FliK were measured by immunoblotting with polyclonal anti-FlgD (second row), anti-FlgE (third row) and anti-Flik (fourth row) antibodies, respectively. The asterisk indicates degradation products of wild-type FlgD that were presumably due to a relatively unstable conformation of the $\mathrm{N}$-terminal domain of FlgD. 
To further confirm this, we looked for partially or weakly motile $f \lg D$ mutants with C-terminal truncation. We screened 15 spontaneous flgD mutants of Salmonella isolated by the laboratories of S. Yamaguchi and R. M. Macnab (Yale University, New Haven, CT, USA) for motility in soft agar plates in comparison with a wild-type strain and a $f l g D_{(1-72 f-s+1 a a)}$ mutant. We identified two weakly motile flgD mutant strains, MMD623 and MMD651 (Fig. 3c). The mutation identified in MMD623 was a nonsense mutation in the flgD allele that generated an amber (TAG) stop codon at position 196. The other mutation in MMD651 was a single-base insertion between base pairs at A-415 and C-416, causing a frameshift mutation in FlgD. Thus, these mutants produce Cterminally truncated variants of $\mathrm{FlgD}, \mathrm{FlgD}_{(1-195)}$ and $\operatorname{FlgD}_{(1-138 f-s+4 a a)}$ (Fig. 2b).

We examined whether these C-terminal truncations affected the stability or secretion of FlgD (Fig. 3d, second row). We found that immunodetection of purified $\mathrm{FlgD}_{(1-138 f-s+4 a a)}$ by our polyclonal anti-FlgD antibody was much weaker than that of purified $\mathrm{FlgD}$ and $\mathrm{FlgD}_{(1-195)}$ (data not shown), indicating that the polyclonal anti-FlgD antibody efficiently recognizes the C-terminal portion of FlgD. Wild-type $\mathrm{FlgD}, \mathrm{FlgD}_{(1-195)}$ and $\mathrm{FlgD}_{(1-138 f-s+4 a a)}$ were detected in the whole-cell fractions (lanes 1, 3 and 4), whereas $\mathrm{FlgD}_{(1-72 \mathrm{f}-\mathrm{s}+1 \mathrm{laa})}$ was not (lane 2). These results indicate that the poor motility of the $f \lg D_{(1-195)}$ and $f \lg D_{(1-138 f-s+4 a a)}$ mutants is not due to the instability of the proteins encoded by these mutant $f \lg D$ alleles. In the culture supernatants, wild-type $\mathrm{FlgD}, \quad \mathrm{Flg}_{(1-195)}$ and $\mathrm{FlgD}_{(1-138 f-s+4 a a)}$ were observed (lanes 5,7 and 8 ), while $\mathrm{FlgD}_{(1-72 \mathrm{f}-\mathrm{s}+\text { laa) }}$ was not (lane 6). The lack of detection of
$\mathrm{FlgD}_{(1-72 f-s+1 a a)}$ may be due to protein instability or the lack of epitopes recognized by the polyclonal anti-FlgD antibody.

Like the $f l g D_{(1-72 f-s+1 a a)}$ and $f l g D_{(1-86)}$ mutants, the $f l g D_{(1-195)}$ and $f l g D_{(1-138 f-s+4 a a)}$ mutants also secreted higher amounts of FlgE into the culture medium than wild-type cells (Fig. 3d, third row). This indicates that these mutant FlgD proteins cannot prevent most FlgE monomers from leaking out. Interestingly, the $f l g D_{(1-195)}$ and $f l g D_{(1-138 f-s+4 a a)}$ mutants secreted FliC, FlgK and FlgL into the culture medium at almost wild-type levels (Fig. 3d, first row). As the export of FliC, FlgK and FlgL occurs only after completion of hook assembly (Minamino et al., 2008), these mutants produce the hooks and hence switch the export specificity of the export apparatus.

\section{Hook length and morphology}

It has been shown that inefficient hook polymerization considerably affects hook length (Moriya et al., 2006). Therefore, we prepared hook basal bodies from wild-type cells and the $f \lg D_{(1-195)}$ and $f \lg D_{(1-138 f-s+4 a a)}$ mutants, and measured their hook length. The means \pm SDs were $38 \pm 15 \mathrm{~nm}$ for the $f l g D_{(1-195)}$ mutant and $42 \pm 12 \mathrm{~nm}$ for the $f \lg D_{(1-138 f-s+4 a a)}$ mutant, compared with $52 \pm 8 \mathrm{~nm}$ for the wild-type (Fig. 4). While the majority of wild-type hooks had lengths rather sharply distributed within a range from 45 to $60 \mathrm{~nm}$ (Fig. $4 \mathrm{a}$ ), the $f l g D_{(1-195)}$ mutant produced many short hooks distributed over a range between 5 and $45 \mathrm{~nm}$, although the peak of the length distribution was still around 45-50 nm (Fig. 4b). Although the broadening of the hook length distribution was less prominent for the $f l g D_{(1-138 f-s+4 a a)}$ mutant, this mutant also produced a significant fraction (a)


(b)


(c)
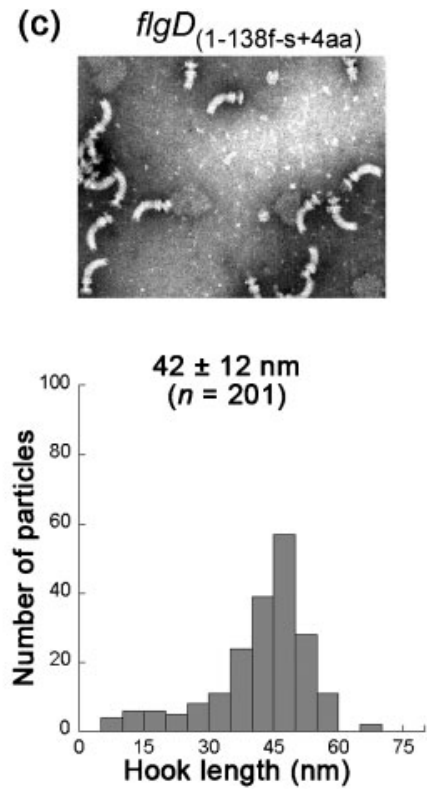

Fig. 4. Electron micrographs of the hook basal bodies with the hook length distributions. (a) SJW1103 (wild-type; WT), (b) MMD623 $\left[f / g D_{(1-195)}\right]$ and (c) MMD651 $\left[f / g D_{(1-138 f-s+4 a a)}\right]$. 
of short hooks with a length distributed over a range between 5 and $45 \mathrm{~nm}$, while the peak population was still between 45 and $50 \mathrm{~nm}$ (Fig. 4c). These results suggest that the hook polymerization ability of these flgD mutants is lower than that of wild-type cells.

It has been shown that any lowering of the efficiency of hook polymerization results in an increase in the secretion level of rod/hook-type export substrates because the rod/hook-type export mode of the flagellar protein export apparatus continues for a longer period (Moriya et al., 2006). The hook length control protein FliK is one of the rod/hook-type substrates (Minamino et al., 1999). Therefore, we tested whether the $\mathrm{C}$-terminal truncations of FlgD also affected the secretion of FliK. Immunoblotting with polyclonal anti-FliK antibody revealed that the secretion level of FliK was much higher than that of the wild-type in the supernatant fractions of the $f \lg D_{(1-195)}$ and $f \lg D_{(1-138 f-s+4 a a)}$ mutants, and slightly less than that of the $f \lg D_{(1-72+1 \text { aa) }}$ mutant (Fig. 3d, fourth row), supporting the idea that their hook polymerization ability is significantly reduced.

\section{Multicopy effect of FlgE on motility of the flgD mutants}

We investigated whether overproduction of FlgE could result in partial restoration of motility of a $f \lg D_{(1-138 \mathrm{f}-\mathrm{s}+4 \mathrm{aa})}$ mutant. Wild-type FlgD complemented the $f \lg D_{(1-72+1 \text { aa) }}$ and $f \lg D_{(1-138 f-s+4 a a)}$ mutants (Fig. 5a), indicating that these mutant alleles are recessive. Consistently, these alleles did not inhibit wild-type motility (data not shown).
Overexpression of FlgE, which increased both cellular and secretion levels of FlgE (data not shown), restored motility of the $f \lg D_{(1-138 f-s+4 a a)}$ mutant to some degree, but not that of cells of either the wild-type or the $f \lg D_{(1-72+1 \text { aa })}$ mutant (Fig. 5a). We obtained the same results with a $f l g D_{(1-195)}$ mutant (data not shown).

To investigate how motility of the $f \lg D_{(1-138 f-s+4 a a)}$ mutant would be improved by overproduction of FlgE, we prepared the hook basal bodies produced by the $f \lg D_{(1-138 f-s+4 a a)}$ mutant overexpressing FlgE and measured the hook length (Fig. 5b). The mean \pm SD of the hook length of the mutant was $52 \pm 19 \mathrm{~nm}$. The peak position was nearly recovered to that of the wild-type, indicating that overproduction of FlgE increases the efficiency of hook assembly in the $f l g D_{(1-138 f-s+4 a a)}$ mutant. This suggests that the $\mathrm{FlgD}_{(1-138 f-s+4 a a)}$ cap cannot efficiently promote the assembly of newly exported FlgE monomers at the hook tip. In agreement with previous observations that wild-type cells overproducing FlgE produce longer hooks with a broader length distribution than the wild-type (Muramoto et al., 1999; Moriya et al., 2006), the SD of the length distribution was still much larger than the wild-type value (Fig. 5b). This indicates that hook length is not well controlled.

\section{DISCUSSION}

Most of the component proteins of the flagellar axial structure require capping proteins to assemble at the distal end of the growing structure. The filament cap, (a)

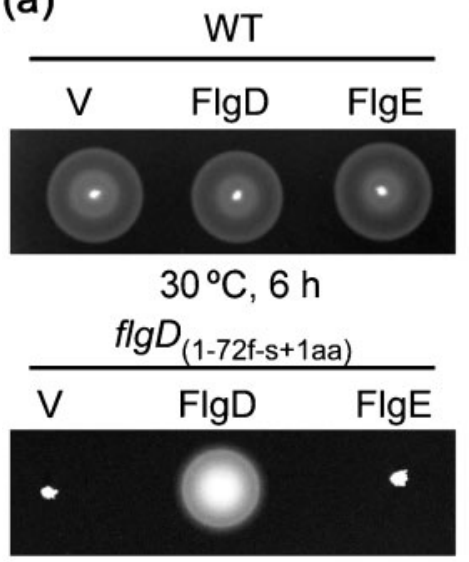

$30^{\circ} \mathrm{C}, 5.5 \mathrm{~h}$

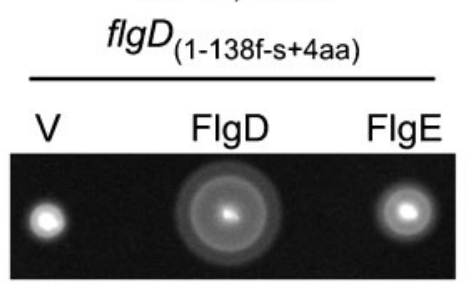

$30^{\circ} \mathrm{C}, 5.5 \mathrm{~h}$ (b)
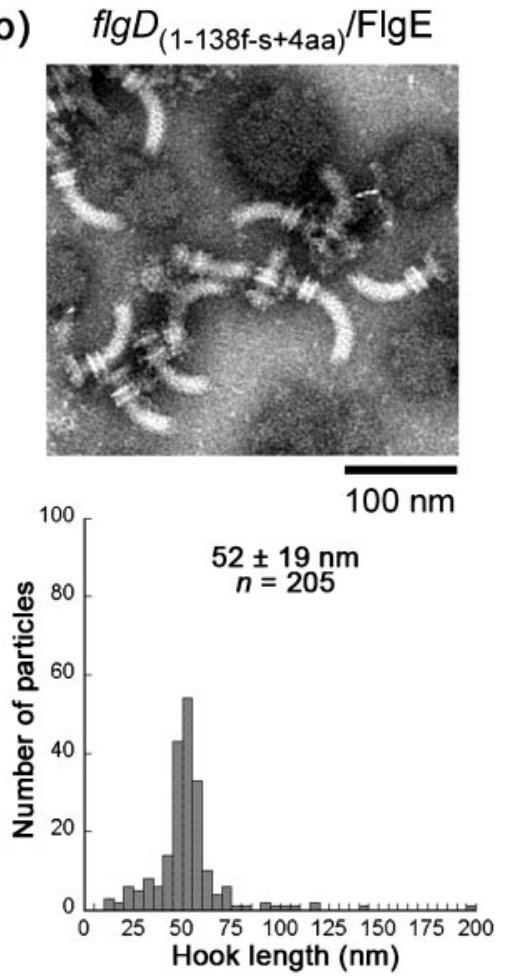

Fig. 5. Multicopy effects of wild-type FlgE on motility and hook length distribution of the Cterminally truncated $f / g D$ mutant variants. (a) Motility of SJW1103 (wild-type; WT), SJW156 $\left[f / g D_{(1-72 f-s+1 a a)}\right]$ and MMD651 $\left[f l g D_{(1-138 f-s+4 a a)}\right]$ transformed with pTrc99A (V), pNM001 (FlgE) or pRCD001 (FlgD) in soft agar plates. (b) Electron micrographs of the hook basal bodies with the hook length distribution of MMD651 $\left.\left[f / g D_{(1-138 f-s}+4 a a\right)\right]$ carrying pNM001 (FlgE). 
which is formed by the FliD protein, has five axially extended leg-like domains that bind to the distal end of the filament to promote filament formation by the assembly of FliC. The leg-like domains of the FliD cap, which is composed of disordered $\mathrm{N}$ - and C-terminal regions of FliD, play an important role in creating an open assembly site for newly exported FliC subunits (Yonekura et al., 2000).

FlgD acts as the hook cap to allow newly exported FlgE monomers to polymerize at the distal end of the hook (Ohnishi et al., 1994). The flexible N-terminal domain of $\mathrm{FlgD}$ is sufficient for the hook-capping function (Kutsukake \& Doi, 1994). Like the filament, the hook is a tubular structure made of 11 protofilaments (Fujii et al., 2009). Therefore, it is plausible that $\mathrm{Flg}_{\mathrm{N}}$ may form flexible leg-like domains that bind to the hook tip in a way similar to the FliD cap. However, little is known about the role of $\mathrm{FlgD}_{\mathrm{C}}$ in hook assembly. To clarify this, we isolated pseudorevertants from flgE point mutants that were severely hook assembly-deficient at $42{ }^{\circ} \mathrm{C}$. Interestingly, most of the second-site mutations were located within $\mathrm{FlgD}_{\mathrm{C}}$ (Fig. 2). These second-site $f \lg D$ mutations blocked leakage of mutant $\mathrm{FlgE}$ subunits into the culture medium to some degree (Fig. 2c), thereby making hook assembly relatively more efficient. The parental $f l g E$ mutations are not located on the subunit interface in the hook structure (Fig. 1d). The second-site $f l g D$ mutations displayed allele specificity. Therefore, we suggest that the second-site flgD mutations compensate for the first-site $f l g E$ mutations by altering the physical interface between FlgD and FlgE for hook polymerization. When the second-site mutations are mapped on the crystal structure of $\mathrm{FlgD}_{\mathrm{C}}$ from $X$. campestris, which contains residues 84-221 (Kuo et al., 2008), interestingly, they are localized on one side of the molecular surface of $\mathrm{FlgD}_{\mathrm{C}}$ (Fig. 6). This raises the possibility that $\mathrm{FlgD}_{\mathrm{C}}$ interacts with newly exported FlgE subunits through this surface, and the interaction between $\mathrm{FlgD}_{\mathrm{C}}$ and $\mathrm{FlgE}$ may contribute to efficient and proper incorporation of the FlgE subunit into the growing hook.

We next characterized two weakly motile $f \lg D$ mutants encoding C-terminally truncated variants, $\mathrm{FlgD}_{(1-195)}$ and $\mathrm{FlgD}_{(1-138 f-\mathrm{s}+4 \mathrm{aa})}(\mathrm{Fig} .3 \mathrm{c})$. The levels of FlgE secreted by these $f l g D$ mutants were much higher than those secreted by wild-type cells and were similar to those of an $f l g D$ null mutant that does not form the hook (Fig. 3d). Unlike the $f l g D$ null mutant, they retained the ability to form the hook to a considerable degree (Fig. 4). However, many of the hooks produced by these mutants had lengths broadly distributed towards ones shorter than those produced by the wild-type cells (Fig. 4). These results indicate that these C-terminally truncated variants of FlgD cannot fully exert the hook-capping function to prevent exported FlgE monomers from leaking out during hook assembly. Therefore, it is very likely that $\mathrm{FlgD}_{\mathrm{C}}$ supports or stabilizes the function of $F \lg D_{\mathrm{N}}$.

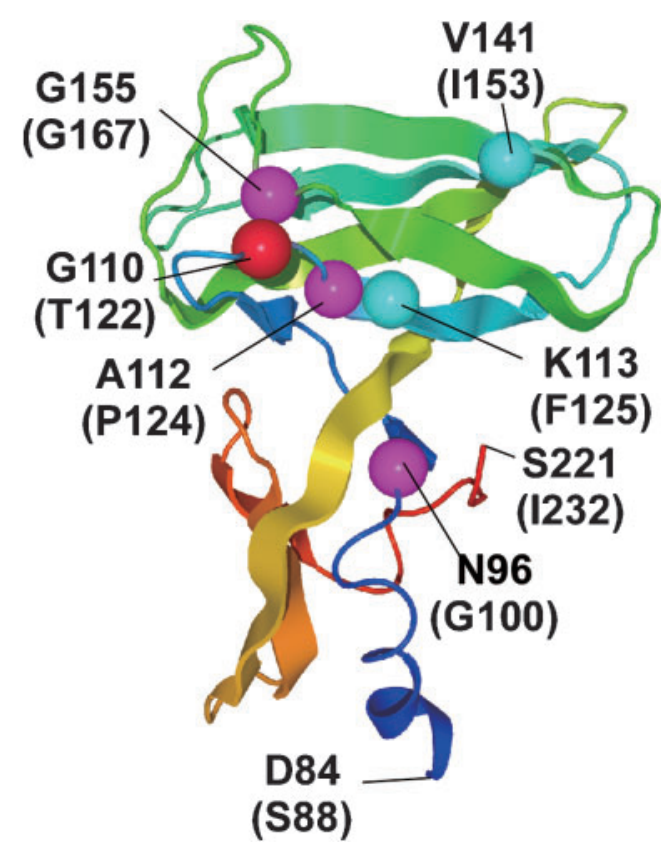

Fig. 6. Atomic model of Xanthomonas FlgD $\mathrm{C}_{\mathrm{C}}$ (PDB ID: 3C12) and locations of the second-site mutations. The mutation sites are shown by balls: magenta, flgE(T149l); red, flgE(T149I) and flgE(R96S); cyan, flgE(I137S). The residue labels for Salmonella and Xanthomonas are shown with and without parentheses, respectively.

The crystal structure of Xanthomonas $\mathrm{FlgD}_{\mathrm{C}}$ shows two compactly folded domains (Kuo et al., 2008). The remaining $\mathrm{N}$-terminal 83 residues, which are unfolded in solution, bind to the distal end of the rod or the hook to anchor $\operatorname{FlgD}_{\mathrm{C}}$, perhaps to be a half-open lid in a similar manner to the pentagonal plate domain of the FliD pentamer cap (Yonekura et al., 2000). Interestingly, the sequence alignment of FlgD proteins from S. enterica and X. campestris (Kuo et al., 2008; Supplementary Fig. S1) suggests that the folded domain structure of $\mathrm{FlgD}_{\mathrm{C}}$ is completely lost in $\operatorname{FlgD}_{(1-86)}$ and $\operatorname{FlgD}_{(1-138 f-s+4 a a)}$. Considering the results of our genetic and functional analyses, the physical trapping of FlgE monomers under the putative half-open lid formed by $\mathrm{FlgD}_{\mathrm{C}}$ for refolding and binding of newly exported FlgE would be one of the roles of the compactly folded domains of $\mathrm{FlgD}_{\mathrm{C}}$. Preliminary X-ray analysis of full-length FlgD derived from Pseudomonas aeruginosa has been carried out (Luo et al., 2009), but its atomic structure is not yet available. In the future, it will be interesting to see extragenic flgE suppressor mutations in the complete FlgD atomic model to understand how these suppressors affect the hook polymerization efficiency. Furthermore, efforts toward high-resolution structures not only of the $\mathrm{FlgD}$ cap structure but also of the FlgD-hook complex will be required for building a model of the hook-capping mechanism. 


\section{ACKNOWLEDGEMENTS}

We acknowledge M. Kihara for technical assistance, continuous support and encouragement. N. M. was a research fellow of the Japan Society for the Promotion of Science. This work was supported in part by Grants-in-Aid for Scientific Research from the Ministry of Education, Culture, Sports, Science and Technology of Japan to T. M., K. I. and K. N.

\section{REFERENCES}

Aizawa, S.-I., Dean, G. E., Jones, C. J., Macnab, R. M. \& Yamaguchi, S. (1985). Purification and characterization of the flagellar hook-basal body complex of Salmonella typhimurium. J Bacteriol 161, 836-849.

Erhardt, M., Hirano, T., Su, Y., Paul, K., Wee, D. H., Mizuno, S., Aizawa, S.-I. \& Hughes, K. T. (2010). The role of the FliK molecular ruler in hook-length control in Salmonella enterica. Mol Microbiol 75, 1272-1284

Fujii, T., Kato, T. \& Namba, K. (2009). Specific arrangement of $\alpha$-helical coiled coils in the core domain of the bacterial flagellar hook for the universal joint function. Structure 17, 1485-1493.

Hirano, T., Yamaguchi, S., Oosawa, K. \& Aizawa, S.-I. (1994). Roles of FliK and FlhB in determination of flagellar hook length in Salmonella typhimurium. J Bacteriol 176, 5439-5449.

Hirano, T., Minamino, T. \& Macnab, R. M. (2001). The role in flagellar rod assembly of the N-terminal domain of Salmonella FlgJ, a flagellum-specific muramidase. J Mol Biol 312, 359-369.

Hirano, T., Minamino, T., Namba, K. \& Macnab, R. M. (2003). Substrate specificity classes and the recognition signal for Salmonella type III flagellar export. J Bacteriol 185, 2485-2492.

Ikeda, T., Asakura, S. \& Kamiya, R. (1985). "Cap" on the tip of Salmonella flagella. J Mol Biol 184, 735-737.

Kato, S., Aizawa, S.-I. \& Asakura, S. (1982). Reconstruction in vitro of the flagellar polyhook from Salmonella. J Mol Biol 161, 551-560.

Kubori, T., Shimamoto, N., Yamaguchi, S., Namba, K. \& Aizawa, S.-I. (1992). Morphological pathway of flagellar assembly in Salmonella typhimurium. J Mol Biol 226, 433-446.

Kuo, W. T., Chin, K. H., Lo, W. T., Wang, A. H. \& Chou, S. H. (2008), Crystal structure of the C-terminal domain of a flagellar hookcapping protein from Xanthomonas campestris. J Mol Biol 381, 189199.

Kutsukake, K. \& Doi, H. (1994). Nucleotide sequence of the flgD gene of Salmonella typhimurium which is essential for flagellar hook formation. Biochim Biophys Acta 1218, 443-446.

Luo, M., Niu, S., Yin, Y., Huang, A. \& Wang, D. (2009). Cloning, purification, crystallization and preliminary X-ray studies of flagellar hook scaffolding protein FlgD from Pseudomonas aeruginosa PAO1. Acta Crystallogr Sect F Struct Biol Cryst Commun 65, 795-797.
Macnab, R. M. (2003). How bacteria assemble flagella. Annu Rev Microbiol 57, 77-100.

Minamino, T. \& Macnab, R. M. (1999). Components of the Salmonella flagellar export apparatus and classification of export substrates. J Bacteriol 181, 1388-1394.

Minamino, T. \& Macnab, R. M. (2000). Domain structure of Salmonella FlhB, a flagellar export component responsible for substrate specificity switching. J Bacteriol 182, 4906-4914.

Minamino, T. \& Namba, K. (2004). Self-assembly and type III protein export of the bacterial flagellum. J Mol Microbiol Biotechnol 7, 5-17.

Minamino, T., González-Pedrajo, B., Yamaguchi, K., Aizawa, S.-I. \& Macnab, R. M. (1999). FliK, the protein responsible for flagellar hook length control in Salmonella, is exported during hook assembly. Mol Microbiol 34, 295-304.

Minamino, T., Imada, K. \& Namba, K. (2008). Mechanisms of type III protein export for bacterial flagellar assembly. Mol Biosyst 4, 11051115.

Minamino, T., Moriya, N., Hirano, T., Hughes, K. T. \& Namba, K. (2009). Interaction of FliK with the bacterial flagellar hook is required for efficient export specificity switching. Mol Microbiol 74, 239-251.

Moriya, N., Minamino, T., Hughes, K. T., Macnab, R. M. \& Namba, K. (2006). The type III flagellar export specificity switch is dependent on FliK ruler and a molecular clock. J Mol Biol 359, 466-477.

Muramoto, K., Makishima, S., Aizawa, S.-I. \& Macnab, R. M. (1999). Effect of hook subunit concentration on assembly and control of length of the flagellar hook of Salmonella. J Bacteriol 181, 5808-5813.

Ohnishi, K., Ohto, Y., Aizawa, S.-I., Macnab, R. M. \& lino, T. (1994). $\mathrm{FlgD}$ is a scaffolding protein needed for flagellar hook assembly in Salmonella typhimurium. J Bacteriol 176, 2272-2281.

Saijo-Hamano, Y., Minamino, T., Macnab, R. M. \& Namba, K. (2004). Structural and functional analysis of the C-terminal cytoplasmic domain of FlhA, an integral membrane component of the type III flagellar protein export apparatus in Salmonella. J Mol Biol 343, 457466

Samatey, F. A., Matsunami, H., Imada, K., Nagashima, S., Shaikh, T. R., Thomas, D. R., Chen, J. Z., Derosier, D. J., Kitao, A. \& Namba, K. (2004). Structure of the bacterial flagellar hook and implication for the molecular universal joint mechanism. Nature 431, 1062-1068.

Yamaguchi, S., Fujita, H., Sugata, K., Taira, T. \& lino, T. (1984). Genetic analysis of $H 2$, the structural gene for phase-2 flagellin in Salmonella. J Gen Microbiol 130, 255-265.

Yonekura, K., Maki, S., Morgan, D. G., DeRosier, D. J., Vonderviszt, F., Imada, K. \& Namba, K. (2000). The bacterial flagellar cap as the rotary promoter of flagellin self-assembly. Science 290, 2148-2152.

Edited by: P. W. O'Toole 